Pacific Journal of Mathematics

SOME MAPPINGS WHICH DO NOT ADMIT AN AVERAGING 


\title{
SOME MAPPINGS WHICH DO NOT ADMIT AN AVERAGING OPERATOR
}

\author{
John WARREN BAKer AND R. C. LACHER
}

\begin{abstract}
The problem of determining for spaces $X$ and $Y$ necessary and sufficient conditions such that there exists a map $\phi$ of $X$ onto $Y$ which does not admit an averaging operator is considered. This corresponds to identilying the uncomplemented closed selfadjoint subalgebras of $C(X)$ which contain $1_{x}$. Mappings $\phi$ of $X$ onto $Y$ are constructed which do not admit averaging operators, for example, when $X$ is any uncountable compact metric space and $Y$ is any countable product of intervals. Also, $X$ can be any space containing an open set homeomorphic to a Banach space and $Y=X$. These results generalize earlier work by D. Amir and S. Ditor.
\end{abstract}

If $\phi$ is a mapping of $X$ onto $Y$, the induced operator $\phi^{0}$ from $C(Y)$ to $C(X)$ that takes $f \in C(Y)$ to $f \circ \phi \in C(X)$ is a multiplicative isometric isomorphism. In case $\phi$ is a quotient map (e.g., if $X$ and $Y$ are compact Hausdorff spaces) then $\phi^{0}(C(Y))$ consists of all functions in $C(X)$ which are constant on each point inverse of $\phi$. We say $\phi$ admits an averaging operator if there is a projection of $C(X)$ onto $\phi^{0}(C(Y))$. It is easily seen that $\phi$ admits an averaging operator if and only if there exists a bounded linear operator $u$ from $C(X)$ into $C(Y)$ such that $u \phi^{0}(f)=f$ for each $f \in C(Y)$ (see [12], Cor. 3.2), and in this case $u$ is called an averaging operator for $\phi$.

Following the appearance of the monograph by A. Pelczynski on averaging and extension operators [12], there has been much interest in the study of averaging operators (e.g., see [2], [3], [4], [5], [6], [15]). A central problem in this study, known as the complemented subalgebra problem, is to determine necessary and sufficient conditions for a map $\phi$ from a compact Hausdorff space $X$ onto a compact Hausdorff space $Y$ to admit an averaging operator. Strong necessary conditions have been established in [5]. (Also, see [2] and [3].) Two closely related problems are to determine for compact Hausdorff spaces $X$ and $Y$ necessary and sufficient conditions that there exists a map $\phi$ of $X$ onto $Y$ which (1. admits; 2. does not admit) an averaging operator. Since this corresponds to determining the complemented and uncomplemented closed selfadjoint subalgebras of $C(X)$ which contain $1_{X}$ by Stone's Theorem [14, p. 122], results of this type yield information about the structure of $C(X)$.

In $1968, \mathrm{~S}$. Ditor established that there is a map $\phi$ of $[0,1]$ onto itself 
which does not admit an averaging operator (see [6] and also [5]). In [3], it was shown that if a topological space $X$ contains an open 0 -dimensional compact metric space $K$ with $K^{(\omega)}$ nonempty, then there is a map $\phi$ of $X$ onto itself which does not admit an averaging operator. The same result was also established if $K$ is a first-countable compact subset of $X$ and $\operatorname{Int}(K)^{(n)}$ contains an isolated point for each integer $n$. It has recently been shown [4] that if $X$ and $Y$ are compact metric spaces with $\left|X^{(\alpha)}\right| \geqq\left|Y^{(\alpha)}\right|$ for each ordinal number $\alpha, X$ is 0 -dimensional, and $Y^{(\omega)}$ is nonempty, there is a map $\phi$ of $X$ onto $Y$ which does not admit an averaging operator. (Also, see [4] for other related results.)

All of the preceding results except the one by Ditor require the space $X$ to be 0 -dimensional. In this paper, we continue this study by considering Hausdorff spaces $X$ and $Y$ which are not necessarily 0 -dimensional and establishing sufficient conditions such that there will exist a map $\phi$ of $X$ onto $Y$ which does not admit an averaging operator. For example, we show that if $X$ is locally a Banach space at some point, then there is a map $\phi$ of $X$ onto itself which does not admit an averaging operator (Theorem 2). The same conclusion holds if $X=I^{\alpha}$ for any cardinal number $\alpha \geqq 1$ (Corollary 1.1). Another corollary is that if $X$ is any nondispersed compact Hausdorff space and $Y$ is any cube $I^{\alpha}, 1 \leqq \alpha \leqq \boldsymbol{N}_{0}$, then there exists a map $\phi$ of $X$ onto $Y$ which does not admit an averaging operator (Corollary 3.1). These results generalize the previously mentioned result by Ditor and the well-known theorem by $\mathrm{D}$. Amir [1] that $C[0,1]$ contains an uncomplemented subspace isometrically isomorphic to $C[0,1]$.

The terminology used herein is standard and follows that in Dunford and Schwartz's Linear Operators I [9] and Dugundji's Topology [7]. We let $I=[0,1]$.

Let $S$ be a topological space. The cone $K$ over $S$ is the quotient space $(I \times S) / R$ where $R$ is the equivalence relation $(0, x) \sim\left(0, x^{\prime}\right)$ for all $x, x^{\prime} \in S$ (see [7, p. 126]). The vertex of this cone is $v=\{0\} \times S$ and $S$ is identified with the base $\{1\} \times S$. Let $Y=I \times K$ and $\dot{Y}=$ $(\{0,1\} \times K) \cup(I \times S)$. Frequently, $\dot{Y}$ is the boundary of $Y$. The preceding assumptions about $Y$ are satisfied by many topological spaces. For example, the closed unit ball $K=\{x \in B \mid\|x\| \leqq 1\}$ in a Banach space $B$ is the cone on the unit sphere $S=\{x \in B\|x\|=1\}$ and the cone on the cube $I^{\alpha}$ for $\alpha \geqq 0$ is homeomorphic to $I^{\alpha+1}\left(\alpha\right.$ finite) or $I^{\alpha}$ $(\alpha$ infinite).

THEOREM 1. There exists a map $\phi$ of Y onto itself such that $\phi(y)=y$ for each $y \in \dot{Y}$ and $\phi$ does not admit an averaging operator.

Proof. Let $\phi_{0}: I \rightarrow I$ be a monotone map such that $\phi_{0}(0)=0$ and $\phi_{0}(1)=1$. Define a map $\tilde{\phi}$ from $I \times I \times S$ onto itself by 


$$
\tilde{\phi}\left(t, t^{\prime}, s\right)=\left(t t^{\prime}+\left(1-t^{\prime}\right) \phi_{0}(t), t^{\prime}, s\right)
$$

and let

$$
\phi: I \times K \rightarrow I \times K
$$

be the map induced by $\tilde{\phi}$ on the quotient space. We claim that $\phi$ maps $I \times(K-\{v\})$ bijectively to itself and that $\phi \mid \dot{Y}$ is the identity. The second statement is obvious. For the first, suppose $\left(t_{1}, t_{1}^{\prime}, s_{1}\right)$ and $\left(t_{2}, t_{2}^{\prime}, s_{2}\right)$ are two points of $I \times I \times S$ with $t_{1}^{\prime}>0$ such that

$$
\tilde{\phi}\left(t_{1}, t_{1}^{\prime}, s_{1}\right)=\tilde{\phi}\left(t_{2}, t_{2}^{\prime}, s_{2}\right) \text {. }
$$

Then $t_{1}^{\prime}=t_{2}^{\prime}, s_{1}=s_{2}$, and

$$
t_{1}-t_{2}=\frac{1-t_{1}^{\prime}}{t_{1}^{\prime}}\left[\phi_{0}\left(t_{2}\right)-\phi_{0}\left(t_{1}\right)\right]
$$

Thus, $t_{1} \neq t_{2}$ implies $\phi_{0}\left(t_{1}\right) \neq \phi_{0}\left(t_{2}\right)$. The claim now follows from the fact that $\phi_{0}$ is monotone, for if $t_{1}<t_{2}$, then $\phi_{0}\left(t_{1}\right)<\phi_{0}\left(t_{2}\right)$; hence,

$$
t_{1} t_{1}^{\prime}+\left(1-t_{1}^{\prime}\right) \phi_{0}\left(t_{1}\right)<t_{2} t_{2}^{\prime}+\left(1-t_{2}^{\prime}\right) \phi_{0}\left(t_{2}\right)
$$

and $\tilde{\phi}\left(t_{1}, t_{1}^{\prime}, s_{1}\right) \neq \tilde{\phi}\left(t_{2}, t_{2}^{\prime}, s_{2}\right)$, a contradiction.

Next, define $E: C(I) \rightarrow C(Y)$ by

$$
E f(t, x)=f(t)
$$

for $(t, x) \in I \times K$. Then $E$ is a linear operator with $\|E\|=1$ and $R E$ is the identity operator on $C(I)$ where $R: C(Y) \rightarrow C(I)$ is the restriction operator with $R f(t)=f(t, v)$. Moreover, since the nondegenerate point inverses of $\phi$ all lie in $I \times v$ (where they are of the form $\phi_{0}^{-1}(t) \times v$ ) it is clear that if $f \in C(I)$ and $f$ is constant on each $\phi_{0}^{-1}(t)$ for each $t \in I$, then $E(f)$ is constant on each $\phi^{-1}(t, x)$ for $(t, x) \in I \times K$. Equivalently, $E\left(\phi_{0}^{0}[C(I)]\right) \subset \phi^{0}[C(Y)]$.

Let $\phi_{0}$ be a map such that $\phi_{0}^{0}[C(I)]$ is uncomplemented in $C(I)$. For example, if $\psi$ is the Cantor map from the Cantor set $\mathscr{C}$ onto $I$ defined by $\psi\left(\sum_{i=1}^{\infty} 2 \xi_{i} / 3^{i}\right)=\sum_{i=1}^{\infty} \xi_{l} / 2^{i}$, then $\phi_{0}$ can be selected to be the map of $I$ onto itself which extends $\psi$ and is constant on the disjoint intervals of $I-\mathscr{C}$ (see [5, Cor. 5.8]). Then either by Corollary 5.5 in [5] or Corollary 1.4 in [2], $\phi_{0}$ does not admit an averaging operator.

Suppose $P$ is a bounded projection of $C(Y)$ onto $\phi^{0}[C(Y)]$. Define $P_{0}: C(I) \rightarrow C(I)$ by $P_{0}=R P E$. Then $P_{0}$ is a bounded linear operator and

$$
P_{0}[C(I)]=R P E[C(I)] \subset R \phi^{0}[C(Y)] \subset \phi_{0}^{0}[C(I)]
$$


Moreover, if $f \in \phi_{0}^{0}[C(I)]$, then $E f \in \phi^{0}[C(Y)]$ and $P_{0}(f)=R P E(f)=$ $R E(f)=f$; hence, $P_{0}^{2}=P_{0}$ and $P_{0}$ is a projection of $C(I)$ onto $\phi_{0}^{0}[C(I)]$, which is a contradiction.

Corollary 1.1. Suppose $X=I^{\alpha}$ for some cardinal $\alpha \geqq 1$. Then there exists a map $\phi$ of $X$ onto itself which does not admit an averaging operator.

Proof. $I^{\alpha}=I \times K$ where $K$ is always a cone except when $\alpha=1$, in which case the above-mentioned result of Ditor applies.

Since the next theorem is applicable to a space $X$ which contains an open set homeomorphic to Euclidean $n$-space for $n \geqq 1$, it generalizes the previously mentioned results of Amir and Ditor.

THEOREM 2. Suppose X contains an open set homeomorphic to some (nonzero) Banach space. Then there exists a map $\phi$ of X onto itself which does not admit an averaging operator.

Proof. If $B$ is a Banach space of dimension greater than one, then $B=R \times B_{1}$ where $R$ is the real line and $B_{1}$ is a Banach space. Let $K$ be the unit ball in $B_{1}$. By Theorem 1, there exists a map $\psi$ of $Y=I \times K$ onto itself such that $\psi^{0}[C(Y)]$ is uncomplemented in $C(Y)$ and $\psi$ is the identity on $\dot{Y}$. Since $B$ may be identified with an open set in $X$, we define $\phi: X \rightarrow X$ to be $\psi$ on $B$ and the identity otherwise. (If $B=R$, we simply extend the Cantor function $\psi: I \rightarrow I$ used by Ditor to $\phi: X \rightarrow X$.

Suppose $P$ is a projection of $C(X)$ onto $\phi^{0}[C(X)]$. Since $Y$ is bounded in $B$, there is a closed neighborhood $V$ of $Y$ in $B$. Let $Z=Y \cup(V-$ Int $V)$ and define $T: C(Y) \rightarrow C(Z)$ by $T f(x)=f(x)$ for $x \in Y$ and $T f(x)=0$ otherwise. Then $T$ is a linear operator with $\|T f\|=\|f\|$ (i.e., $T$ is a simultaneous extension operator). By the Borsuk-Dugundji Simultaneous Extension Theorem (see [8, p. 360] or $[13$, p. 37]), there is a linear operator $E: C(Z) \rightarrow C(V)$ with $\|E f\|=\|f\|$ and $E f(x)=f(x)$ for $x \in Z$. Let $M=\{f \in C(V) \mid f(x)=0$ for $x \in(V-$ Int $V)\}$ and define $L: M \rightarrow C(X)$ by $L f(x)=f(x)$ for $x \in V$ and $L f(x)=0$ otherwise. Clearly, $L$ is a simultaneous extension operator with $\|L f\|=\|f\|$. Let $R$ be the restriction operator from $C(X)$ onto $C(Y)$ and define $P_{0}=$ RPLET. Clearly, $P_{0}$ is a linear operator on $C(Y)$ with $\left\|P_{0}\right\|=\|P\|$. Moreover,

$$
P_{0}[C(Y)] \subset R P[C(X)] \subset R \phi^{0}[C(X)] \subset \psi^{0}[C(Y)]
$$

and if $f \in \psi^{0}[C(Y)]$, then $\operatorname{LET}(f) \in \phi^{0}[C(X)]$ and $P_{0}(f)=\operatorname{RPLET}(f)=$ 
$\operatorname{RLET}(f)=f$. Therefore $P_{0}$ is a projection of $C(Y)$ onto $\psi^{0}[C(Y)]$, which is a contradiction.

In the next theorem, we suppose $S$ is a locally connected compact metric space, $K$ is the cone over $S$, and $Y=I \times K$. Recall that a topological space $X$ is called dispersed if $X$ contains no perfect subsets.

THEOREM 3. Suppose $S$ is a locally connected compact metric space and $X$ is a nondispersed compact Hausdorff space (e.g., an uncountable compact metric space). Then there exists a map $\phi$ of $X$ onto $Y$ which does not admit an averaging operator.

Proof. Since $Y$ is a nonempty locally connected continuum, it follows by the Hahn-Mazurkiewicz-Sierpinski Theorem [10, p. 256] that there is a map $\nu$ of $I$ onto $Y$. Since $X$ is nondispersed, there is a map $\psi$ of $X$ onto $I$ [11. Thm. 1]. By Theorem 1, there is a map $\pi$ of $Y$ onto itself such that $\pi^{0}[C(Y)]$ is uncomplemented in $C(Y)$. Let $\phi=$ $\pi \nu \psi$. We show $\phi^{0}[C(Y)]$ is uncomplemented in $C(X)$. Suppose $P$ is a projection of $C(X)$ onto $\phi^{0}[C(Y)]$. If $\lambda=\nu \psi$ and $P_{0}=\left(\lambda^{0}\right)^{-1} P \lambda^{0}$, then $P_{0}$ is a linear operator from $C(Y)$ into $\pi^{0}[C(Y)]$. Moreover, if $f \in$ $\pi^{0}[C(Y)], f=\pi^{0} g$ for some $g \in C(Y)$ and $P_{0}(f)=\left(\lambda^{0}\right)^{-1} P \lambda^{0}\left(\pi^{0} g\right)=$ $\left(\lambda^{0}\right)^{-1} P \phi^{0}(g)=\left(\lambda^{0}\right)^{-1} \phi^{0}(g)=\left(\lambda^{0}\right)^{-1} \lambda^{0}\left(\pi^{0} g\right)=f$. Thus $P_{0}$ is projection of $C(Y)$ onto $\pi^{0}[C(Y)]$, a contradiction.

Since the continuous image of a dispersed space is dispersed [11], we obtain the following characterization.

COROLlary 3.1. Let $1 \leqq n \leqq \boldsymbol{N}_{0}$. If $X$ is a compact Hausdorff space, then there is a map of $X$ onto $I^{n}$ which does not admit an averaging operator if and only if $X$ is not dispersed.

In particular, if $1 \leqq m, n \leqq \boldsymbol{N}_{0}$, then there is a map of $I^{m}$ onto $I^{n}$ which does not admit an averaging operator.

\section{REFERENCES}

1. D. Amir, Continuous function spaces with the bounded extension property, Bull. Res. Counc. of Israel, 10F (1962), 133-138.

2. J. W. Baker, Some uncomplemented subspaces of $C(X)$ of the type $C(Y)$, Studia Math., $\mathbf{3 6}(1970)$, 85-103.

3. Uncomplemented $C(X)$-subalgebras of $C(X)$, Trans. Amer. Math. Soc., 186 (1973), $1-15$.

4. J. W. Baker and J. Wolfe, Averaging operators and $C(X)$-subspaces with the separable projection property, to appear. 
5. S. Ditor, Averaging operators in $C(S)$ and lower semicontinuous sections of continuous maps. Trans. Amer. Math. Soc., 175 (1973), 195-208.

6. Linear operators of averaging and extension, Ph. D. Dissertation written under W. G. Bade, University of California at Berkeley, 1968.

7. J. Dugundji, Topology, Allyn and Bacon, Boston, Mass., 1966.

8. - An extension of Tietze's theorem, Pacific J. Math., 1 (1951), 353-367.

9. N. Dunford and J. Schwartz, Liaear Operators I: General Theory, Pure and Appl. Math., vol. 7, Interscience, New York, 1958.

10. K. Kuratowski, Topologie, vol. II, 3rd ed., Monographie Mat., Tom 21, PWN, Warsaw, 1961; English transl., Academic Press, New York: PWN, Warsaw, 1968.

11. A. Peczynski and Z. Semadeni, Spaces of continuous functions $I I I$, (Spaces $C(\Omega)$ for $\Omega$ without perfect subsets), Studia Math., 18 (1959), 211-222.

12. - Linear extensions, linear averagings, and their applications to classification of continuous function spaces, Dissertations Math. Rozprawy Mat. 58 (1968), 1-92.

13. Z. Semadeni, Simultaneous extensions and projections in spaces of continuous functions, Lecture Notes at Aarhus Universitet, May, 1965.

14. - Banach Spaces of Continuous Functions I, Monografie Matematyczne, Warsaw, Poland, 1971.

15. J. Wolfe, Averaging and extension operators and injective spaces of continuous functions, to appear.

Received December 20, 1973. Alfred P. Sloan Fellow, supported in part by grant NSF-GP-19964.

Kent STATE University

AND

The Florida State University 



\section{Pacific Journal of Mathematics}

\section{Vol. 62, No. $1 \quad$ January, 1976}

Mieczyslaw Altman, Contractor directions, directional contractors and

directional contractions for solving equations . .................. 1

Michael Peter Anderson, Subgroups of finite index in profinite groups .........

Zvi Arad, Abelian and nilpotent subgroups of maximal order of groups of odd order

John David Baildon and Ruth Silverman, On starshaped sets and Helly-type theorems ..........................................

John W. Baker and R. C. Lacher, Some mappings which do not admit an

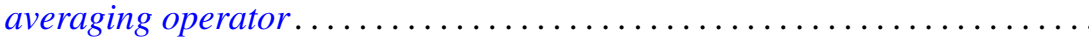

Joseph Barback, Composite numbers and prime regressive isols . . . . . . . . . .

David M. Boyd, Composition operators on $H^{p}(A) \ldots \ldots \ldots \ldots \ldots \ldots \ldots$

Maurice Chacron, Co-radical extension of PI rings . . . . . . . . . . . . .

Fred D. Crary, Some new engulfing theorems . . . . . . . . . . . . . . .

Victor Dannon and Dany Leviatan, A representation theorem for convolution transform with determining function in $L^{p} \ldots \ldots \ldots \ldots \ldots \ldots \ldots \ldots \ldots \ldots \ldots \ldots \ldots \ldots$

Mahlon M. Day, Lumpy subsets in left-amenable locally compact

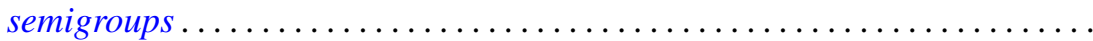

Michael A. Gauger, Some remarks on the center of the universal enveloping algebra of a classical simple Lie algebra . .

David K. Haley, Equational compactness and compact topologies in rings

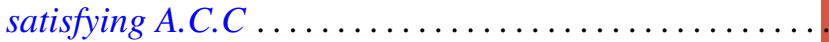

Raymond Heitmann, Generating ideals in Prüfer domains .

Gerald Norman Hile, Entire solutions of linear elliptic equations with

Laplacian principal part. .

Richard Oscar Hill, Moore-Postnikov towers for fibrations in which $\pi_{1}$ (fiber) is non-abelian

John Rast Hubbard, Approximation of compact homogeneous maps . .

Russell L. Merris, Relations among generalized matrix functions . .

V. S. Ramamurthi and Edgar Andrews Rutter, On cotorsion radicals ...

Ralph Tyrrell Rockafellar and Roger Jean-Baptiste Robert Wets, Stochastic

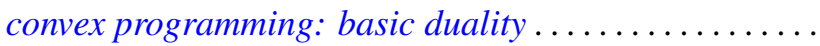

Alban J. Roques, Local evolution systems in general Banach spaces ..

I. Bert Russak, An indirect sufficiency proof for problems with bounded state variables.

Richard Alexander Sanerib, Jr., Ultrafilters and the basis property. .

H. A. Seid, The decomposition of multiplication operators on $L_{p}$-spaces . .

Franklin D. Tall, The density topology .................. 